\title{
Crystal structure of benzyl-(2-hydroxyethyl)-dimethylammonium iodide, $\left[\mathrm{C}_{11} \mathrm{H}_{18} \mathrm{NO}\right]^{+} \mathrm{I}^{-}$
}

\author{
T. Grobosch, S. Sawusch, U. Schilde and E. Uhlemann
}

Universität Potsdam. Institut für Anorganische Chemie und Didaktik der Chemie. Am Neuen Palais 10. PF 161553. D-14415 Potsdam, Germany

Received February 25, 1997, CSD-No. 402867

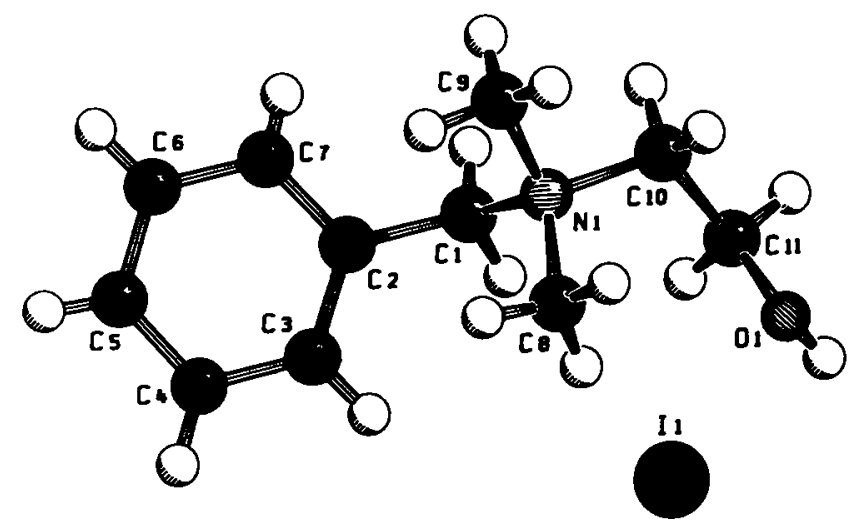

Source of material: The compound was synthesized by reaction of benzyliodide with an ice-cooled solution of 2-(dimethylamino)-ethanol in methanol. After refluxing, the solvent was removed under reduced pressure, and the residue was taken up in ether, filtered and evaporated to a solid.

The title compound is the anchoring group of a strongly basic anion-exchanger of type II.

$\mathrm{C}_{11 \mathrm{H}} \mathrm{HINO}$, monoclinic, $P 12_{1} / a 1$ (No. 14), $a=10.144(9) \AA$ $b=9.994(3) \AA, c=12.511(7) \AA, \beta=94.88(9)^{\circ}, V=1263.8 \AA^{3}, Z=4$, $R(F)=0.027, R_{w}\left(F^{2}\right)=0.062$.
Table 1. Parameters used for the $\mathrm{X}$-ray data collection

\begin{tabular}{|c|c|}
\hline $\begin{array}{l}\text { Crystal: } \\
\text { Wavelength: }\end{array}$ & $\begin{array}{l}\text { white needle, size } 0.08 \times 0.12 \times 0.50 \mathrm{~mm} \\
\text { Mo } K_{\alpha} \text { radiation }(0.71073 \AA)\end{array}$ \\
\hline$\mu:$ & $25.07 \mathrm{~cm}^{-1}$ \\
\hline Diffractometer: & Stoe STADI4 \\
\hline Scan mode: & $\omega / 2 \theta$ \\
\hline$T_{\text {measurement: }}$ & $298 \mathrm{~K}$ \\
\hline $2 \theta_{\max }:$ & $60^{\circ}$ \\
\hline $\mathrm{N}(h k l)_{\text {urique: }}$ & 2176 \\
\hline $\begin{array}{l}\text { Criterion for } I_{0}: \\
N(\text { param })_{\text {refined: }}\end{array}$ & $I_{0}>2 \sigma\left(I_{0}\right)$ \\
\hline $\mathrm{N}(\text { param })_{\text {refined }}$ & 200 \\
\hline Programs: & SHELXS-86, SHELXL-93 \\
\hline
\end{tabular}

Table 2. Final atomic coordinates and displacement parameters (in $\AA^{2}$ )

\begin{tabular}{llllll}
\hline Atom & Site & $x$ & \multicolumn{1}{l}{$y$} & \multicolumn{1}{l}{$z$} & \multicolumn{1}{l}{$U_{\text {iso }}$} \\
\hline H(1A) & $4 e$ & $0.203(4)$ & $-0.145(4)$ & $0.234(3)$ & $0.05(1)$ \\
H(1B) & $4 e$ & $0.315(4)$ & $-0.249(5)$ & $0.225(4)$ & $0.06(1)$ \\
H(3) & $4 e$ & $0.457(4)$ & $-0.299(5)$ & $0.383(4)$ & $0.06(1)$ \\
H(4) & $4 e$ & $0.491(5)$ & $-0.305(5)$ & $0.571(4)$ & $0.09(2)$ \\
H(5) & $4 e$ & $0.347(5)$ & $-0.171(5)$ & $0.667(4)$ & $0.08(2)$ \\
H(6) & $4 e$ & $0.203(5)$ & $-0.033(5)$ & $0.587(4)$ & $0.08(2)$ \\
H(7) & $4 e$ & $0.187(5)$ & $-0.034(5)$ & $0.407(4)$ & $0.06(2)$ \\
H(8B) & $4 e$ & $0.560(4)$ & $-0.025(4)$ & $0.170(3)$ & $0.05(1)$ \\
H(8C) & $4 e$ & $0.542(4)$ & $-0.073(5)$ & $0.287(4)$ & $0.07(2)$ \\
H(8A) & $4 e$ & $0.533(4)$ & $-0.187(5)$ & $0.194(3)$ & $0.05(1)$ \\
H(9B) & $4 e$ & $0.376(4)$ & $0.076(4)$ & $0.307(4)$ & $0.05(1)$ \\
H(9A) & $4 e$ & $0.255(5)$ & $0.090(5)$ & $0.225(3)$ & $0.06(1)$ \\
H(9C) & $4 e$ & $0.389(5)$ & $0.134(5)$ & $0.190(4)$ & $0.06(1)$ \\
H(10A) & $4 e$ & $0.232(4)$ & $-0.050(3)$ & $0.071(3)$ & $0.027(9)$ \\
H(10B) & $4 e$ & $0.372(5)$ & $0.007(5)$ & $0.043(4)$ & $0.06(1)$ \\
H(11B) & $4 e$ & $0.290(4)$ & $-0.200(4)$ & $-0.051(3)$ & $0.05(1)$ \\
H(11A) & $4 e$ & $0.332(4)$ & $-0.275(5)$ & $0.058(4)$ & $0.07(2)$ \\
H(12) & $4 e$ & $0.498(8)$ & $-0.264(8)$ & $-0.042(6)$ & $0.14(3)$ \\
& & & & &
\end{tabular}

Table 3. Final atomic coordinates and displacement parameters (in $\AA^{2}$ )

\begin{tabular}{lllllllllrr}
\hline Atom & Site & $x$ & $y$ & $z$ & $U_{11}$ & $U_{22}$ & $U_{33}$ & \multicolumn{1}{c}{$U_{12}$} & $U_{13}$ & $U_{23}$ \\
\hline $\mathrm{C}(1)$ & $4 e$ & $0.2894(5)$ & $-0.1640(4)$ & $0.2533(3)$ & $0.051(3)$ & $0.035(2)$ & $0.052(2)$ & $-0.005(2)$ & $0.004(2)$ & $0.000(2)$ \\
$\mathrm{C}(2)$ & $4 e$ & $0.3142(4)$ & $-0.1614(4)$ & $0.3730(3)$ & $0.052(2)$ & $0.043(2)$ & $0.044(2)$ & $-0.005(2)$ & $0.005(2)$ & $0.002(2)$ \\
$\mathrm{C}(3)$ & $4 e$ & $0.4061(5)$ & $-0.2449(5)$ & $0.4254(4)$ & $0.081(3)$ & $0.050(3)$ & $0.052(3)$ & $0.011(3)$ & $0.005(2)$ & $0.003(2)$ \\
$\mathrm{C}(4)$ & $4 e$ & $0.4231(6)$ & $-0.2500(6)$ & $0.5366(4)$ & $0.086(4)$ & $0.066(3)$ & $0.055(3)$ & $0.002(3)$ & $-0.005(3)$ & $0.011(3)$ \\
$\mathrm{C}(5)$ & $4 e$ & $0.3453(6)$ & $-0.1701(6)$ & $0.5949(4)$ & $0.091(4)$ & $0.074(4)$ & $0.045(3)$ & $-0.023(3)$ & $0.005(3)$ & $-0.005(3)$ \\
$\mathrm{C}(6)$ & $4 e$ & $0.2542(6)$ & $-0.0857(6)$ & $0.5446(4)$ & $0.083(4)$ & $0.076(4)$ & $0.057(3)$ & $0.000(3)$ & $0.019(3)$ & $-0.009(3)$ \\
$\mathrm{C}(7)$ & $4 e$ & $0.2381(5)$ & $-0.0818(6)$ & $0.4342(4)$ & $0.056(3)$ & $0.064(3)$ & $0.064(3)$ & $0.008(3)$ & $0.015(2)$ & $0.002(3)$ \\
$\mathrm{C}(8)$ & $4 e$ & $0.5155(4)$ & $-0.0936(6)$ & $0.2119(4)$ & $0.045(3)$ & $0.057(3)$ & $0.062(3)$ & $0.001(2)$ & $0.003(2)$ & $-0.003(3)$ \\
$\mathrm{C}(9)$ & $4 e$ & $0.3448(6)$ & $0.0759(5)$ & $0.2298(5)$ & $0.064(3)$ & $0.033(2)$ & $0.066(3)$ & $0.002(2)$ & $0.012(3)$ & $-0.002(2)$ \\
\hline
\end{tabular}


Table 3. (Continued)

\begin{tabular}{|c|c|c|c|c|c|c|c|c|c|c|}
\hline Atom & Site & $x$ & $y$ & $z$ & $U_{11}$ & $U_{22}$ & $U_{33}$ & $U_{12}$ & $U_{13}$ & $U_{23}$ \\
\hline$C(10)$ & $4 e$ & $0.3246(5)$ & $-0.0692(4)$ & $0.0739(3)$ & $0.056(3)$ & $0.043(2)$ & $0.046(2)$ & $0.006(2)$ & $0.006(2)$ & $0.005(2)$ \\
\hline$C(11)$ & $4 e$ & $0.3512(5)$ & $-0.1982(5)$ & $0.0162(4)$ & $0.066(3)$ & $0.045(3)$ & $0.043(2)$ & $-0.005(2)$ & $0.004(2)$ & $-0.006(2)$ \\
\hline $\mathbf{N}(1)$ & $4 e$ & $0.3700(3)$ & $-0.0640(3)$ & $0.1931(3)$ & $0.043(2)$ & $0.032(2)$ & $0.045(2)$ & $0.001(1)$ & $0.005(1)$ & $-0.001(1)$ \\
\hline$O(1)$ & $4 e$ & $0.4835(4)$ & $-0.2009(4)$ & $-0.0090(3)$ & $0.085(3)$ & $0.051(2)$ & $0.086(3)$ & $-0.009(2)$ & $0.039(2)$ & $-0.024(2)$ \\
\hline I(1) & $4 e$ & $0.93956(3)$ & $0.03492(3)$ & $0.18400(2)$ & $0.0602(2)$ & $0.0418(2)$ & $0.0456(2)$ & $0.0002(2)$ & $0.0054(1)$ & $0.0051(1)$ \\
\hline
\end{tabular}

\section{References}

1. Sheldrick, G. M.: SHELXS-86. Program for the solution of crystal structures. University of Göttingen, Germany 1986.

2. Sheldrick, G. M.: SHELXL-93. Program for refining crystal structures. University of Göttingen, Germany 1993. 